\title{
THE KERNEL AND TRACE OPERATORS FOR IDEAL EXTENSIONS OF REGULAR SEMIGROUPS
}

\author{
by MARIO PETRICH
}

(Received 2 November, 1992)

\begin{abstract}
Let $V$ be a regular semigroup and an ideal extension of a semigroup $S$ by a semigroup $Q$. Congruences on $V$ can be represented by triples of the form $(\sigma, P, \tau)$, here called admissible, where $\sigma$ is a congruence on $S, P$ is an ideal of $Q$ and $\tau$ is a 0 -restricted congruence on $Q / P$ satisfying certain conditions. We characterize the trace relation $T$ on $V$ in terms of admissible triples. When the extension $V$ of $S$ is strict, for a congruence $v$ on $V$ given in terms of an admissible triple, we characterize $v_{K}, v^{K}, v_{T}$ and $v^{T}$ again in terms of admissible triples.
\end{abstract}

1. Introduction and summary. Let $S$ be a regular semigroup and $\mathscr{C}(S)$ be its congruence lattice. For $\rho \in \mathscr{C}(S)$, the kernel (respectively, trace) of $\rho$ is the set of all elements of $S \rho$-related to idempotents (respectively, the restriction of $\rho$ to idempotents of $S$ ). The relation on $\mathscr{C}(S)$ which identifies congruences on $S$ with the same kernel (respectively, trace) is the kernel relation $K$ (respectively, the trace relation $T$ ) for $S$. The classes of these (equivalence) relations are intervals and it is convenient to introduce the notation $\rho K=\left[\rho_{K}, \rho^{K}\right]$ and $\rho^{T}=\left[\rho_{T}, \rho^{T}\right]$ for the respective classes of $\rho \in \mathscr{C}(S)$. Then $\rho \rightarrow \rho_{K}$ and $\rho \rightarrow \rho^{K}$ are the kernel operators and $\rho \rightarrow \rho_{T}$ and $\rho \rightarrow \rho^{T}$ are the trace operators. The nature of these operators furnishes valuable information concerning both congruences on $S$ and the congruence lattice $\mathscr{C}(S)$.

Now let $V$ be a regular semigroup with an ideal $S$. Then $V$ is an (ideal) extension of $S$ by $Q=V / S$, where the latter is the Rees quotient semigroup. We may set $V=S \cup Q^{*}$ where $Q^{*}=Q \backslash\{0\}$. In such a case, both $S$ and $Q$ are regular semigroups so the above analysis can be applied to $S, Q$ and $V$. The problem is to reduce this analysis for $V$ to those for $S$ and $Q$. As a first step, we must express the congruences on $V$ in terms of $S$ and $Q$, and if possible, in terms of $\mathscr{C}(S)$ and $\mathscr{C}(Q)$. In the present setting, this problem was solved in [3] as follows. Each congruence on $V$ is expressed in terms of an (admissible) triple $(\sigma, P, \tau)$, where $\sigma \in \mathscr{C}(S), P$ is an ideal of $Q$ and $\tau$ is a 0 -restricted congruence on $Q / P$, satisfying certain conditions.

Representing the congruences on $V$ in terms of triples as above, we may ask whether the relations $K$ and $T$ on $\mathscr{C}(V)$ can be expressed by means of the same relations on $\mathscr{C}(S)$ and $\mathscr{C}(Q)$. We may go one step further by asking for $v_{K}, v^{K}, v_{T}, v^{T}$ for a congruence $v$ on $V$ expressed in terms of a triple. The first task is easy: expressing the kernel and the trace. However, the problem of characterizing $K$ on $\mathscr{C}(V)$ does not seem to admit a convenient solution, whereas $T$ admits a simple expression. The problem with the kernel and the trace operators in this generality does not seem amenable to a successful treatment.

In order to make some progress in this context, we restrict our attention to the special case when $V$ is a strict (or retract) extension of $S$ and in various situations add further restrictions. For strict extensions, we are able to characterize $v_{K}, v^{K}, v_{T}$ and $v^{T}$.

Section 2 contains some terminology, notation, background material and preliminary results. The relation $T$ on $\mathscr{C}(V)$ is characterized in Section 3. In the remaining part of the 
paper, we assume that $V$ is a strict extension of $S$. For a congruence $v$ on $V$ given by means of an admissible triple, we calculate $v_{T}$ and $v^{T}$ in Section 4 and $v_{K}$ and $v^{K}$ in Section 5 again in terms of admissible triples.

2. Preliminaries. In addition to the standard terminology and notation, which can be found, for example, in [1], we state explicitly the following nomenclature and symbolism.

Let $X$ be a set. The equality relation on $X$ is denoted by $\epsilon_{X}$ or simply by $\epsilon$. The universal relation on $X$ is denoted by $\omega_{X}$. The restriction of a function or a relation $\theta$ to $X$ is denoted by $\left.\theta\right|_{X}$. If $\theta$ is an equivalence relation on $X$ and $x \in X$, then $x \theta$ denotes the $\theta$-class containing $x$. If also $A \subseteq X$, then

$$
A \theta=\{x \in X \mid x \theta a \text { for some } a \in A\}
$$

is the saturation of $A$ by $\theta$; if $A \theta=A$, then $\theta$ saturates $A$. If $Y$ is also a set, then $X \backslash Y=\{x \in X \mid x \notin Y\}$.

Let $R$ be a semigroup. If $A \subseteq R$, then $E(A)$ denotes the set of all idempotents in $A$. If $R$ has an identity, then $R^{1}=R$ otherwise $R^{1}$ stands for $R$ with an identity adjoined. The congruence lattice of $R$ is denoted by $\mathscr{C}(R)$. Assume that $R$ has a zero. If $A \subseteq R$, then $A^{*}=A \backslash\{0\}$. An equivalence relation $\theta$ on $R$ is 0 -restricted if $\{0\}$ is a $\theta$-class; the set of all 0 -restricted congruences on $R$ is denoted by $\mathscr{C}_{0}(R)$. Further, $R$ is categorical at zero if for any $a, b, c \in R, a b \neq 0$ and $b c \neq 0$ imply $a b c \neq 0$.

If $\theta$ is a relation on $R$, then $\theta^{*}$ denotes the congruence on $R$ generated by $\theta$. If $\theta$ is an equivalence relation on $R$, then $\theta^{\prime \prime}$ denotes the greatest congruence on $R$ contained in $\theta$; explicitly

$$
a \theta^{\prime \prime} b \text { if } x a y \theta x b y \text { for all } x, y \in R^{1} .
$$

If $A \subseteq Q$, let $\theta$ be the equivalence relation on $R$ whose classes are $A$ and $Q \backslash A$ (whichever one is nonempty), then $\pi_{A}=\theta^{0}$ is the principal congruence relative to $A$; explicitly

$$
a \pi_{A} b \text { if }\left(x a y \in A \Leftrightarrow x b y \in A \text { for all } x, y \in R^{1}\right) .
$$

In fact, $\pi_{A}$ is the greatest congruence $\rho$ on $R$ which saturates $A$. We shall sometimes write $\pi_{A}^{R}$ for emphasis. If $R$ has a zero, then $\zeta_{R}=\pi_{\{0\}}$ is the greatest 0 -restricted congruence on $R$. If $I$ is an ideal of $R$, then $R / I$ denotes the Rees quotient semigroup of $R$ relative to $I$; as a set $R / I=(R \backslash) \cup\{0\}$.

Let $R$ be a regular semigroup, that is for every $a \in R$ there exists $x \in R$ such that $a=a x a$. Let $\rho \in \mathscr{C}(R)$. Then

$$
\operatorname{ker} \rho=\{a \in R \mid a \rho e \text { for some } e \in E(R)\}, \quad \operatorname{tr} \rho=\left.\rho\right|_{E(R)} .
$$

are the kernel and the trace of $\rho$, respectively. They induce a complete $\wedge$-congruence $K$ and a complete congruence $T$ on $\mathscr{C}(R)$ by

$$
\lambda K \rho \text { if } \operatorname{ker} \lambda=\operatorname{ker} \rho, \quad \lambda T \rho \text { if } \operatorname{tr} \lambda=\operatorname{tr} \rho,
$$

respectively. The $K$ - and $T$-classes are intervals, so we use the notation

$$
\rho K=\left[\rho_{K}, \rho^{K}\right], \quad \rho T=\left[\rho_{T}, \rho^{T}\right] .
$$


Lemma 2.1. ([2], Theorem 3.2). Let $R$ be a regular semigroup and $\rho \in \mathscr{C}(R)$. Then

$$
\rho_{K}=\left\{\left(x, x^{2}\right) \mid x \in \operatorname{ker} \rho\right\}^{*}, \quad \rho^{K}=\pi_{\mathrm{ker} \rho} .
$$

We say that $\rho$ is idempotent pure if $\operatorname{ker} \rho=E(S)$.

LEMMA 2.2. ([2], Theorem 3.2). Let $R$ be a regular semigroup, $\rho \in \mathscr{C}(R), \theta=\operatorname{tr} \rho$ and denote by juxtaposition the product of binary relations. Then

$$
\rho_{T}=\theta^{*}, \quad \rho^{T}=(\mathscr{L} \theta \mathscr{L} \theta \mathscr{L} \cap \mathscr{R} \theta \mathscr{R} \theta \mathscr{R})^{0} .
$$

Throughout the paper we fix the following notation: $V$ is a regular semigroup and an (ideal) extension of $S$ by $Q$. Hence $S$ is an ideal of $V$, the Rees quotient $V / S \cong Q$, where we set $V=S \cup Q^{*}$; in addition, both $S$ and $Q$ are regular semigroups.

From ([3, Corollary 1 to Theorem 1]) we deduce the following description of congruences on $V$. Let $\sigma \in \mathscr{C}(S), P$ be an ideal of $Q$ and $\tau \in \mathscr{C}_{10}(Q)$ satisfy the following conditions:

(i) $a, b \in Q \backslash P, a \tau b, x \sigma y \Rightarrow a x \sigma b y, x a \sigma y b$,

(ii) for every $a \in P^{*}$ there exists $a^{\prime} \in S$ such that $x \in S \Rightarrow a x \sigma a^{\prime} x, x a \sigma x a^{\prime}$.

In such a case, we say that $a$ and $a^{\prime}$ are $\sigma$-linked, call $(\sigma, P, \tau)$ an admissible triple and define a relation $v$ on $V$ by

$$
a v b \Leftrightarrow\left\{\begin{array}{lll}
a \tau b & \text { if } & a, b \in Q \backslash P, \\
a^{\prime} \sigma b^{\prime} & \text { if } & a, b \in P^{*}, \\
a^{\prime} \sigma b & \text { if } & a \in P^{*}, b \in S, \\
a \sigma b^{\prime} & \text { if } & a \in S, b \in P^{*} \\
a \sigma b & \text { if } & a, b \in S,
\end{array}\right.
$$

where $a, a^{\prime}$ and $b, b^{\prime}$ are $\sigma$-linked. Then $v$ is a congruence on $V$ and, conversely, every congruence on $V$ has this form. According to ([4, Corollary 3.2]), this representation is unique.

The notation $v=\mathscr{C}(\sigma, P, \tau)$ will always denote the above congruence implicitly implying that $(\sigma, P, \tau)$ is an admissible triple.

In fact, given $v \in \mathscr{C}(V)$, the admissible triple for $v$ is $(\sigma, P, \tau)$, where

$$
\begin{gathered}
\sigma=\left.v\right|_{s}, \quad P=\left\{a \in Q^{*} \mid a v b \text { for some } b \in S\right\} \cup\{0\}, \\
a \tau b \Leftrightarrow a, b \in Q \backslash P, \quad a v b, \text { and } 0 \tau 0 .
\end{gathered}
$$

Note that if $a, a^{\prime}$ are $\sigma$-linked and $\sigma \subseteq \sigma^{\prime}$ for $\sigma^{\prime} \in \mathscr{C}(S)$, then $a, a^{\prime}$ are also $\sigma^{\prime}$-linked. We shall need the following criterion for inclusion of congruences on $V$.

Lemma 2.3. ([4, Lemma 3.1]). Let $v_{i}=\mathscr{C}\left(\sigma_{i}, P_{i}, \tau_{i}\right)$ for $i=1,2$. Then $v_{1} \subseteq v_{2}$ if and only if $\sigma_{1} \subseteq \sigma_{2}, P_{1} \subseteq P_{2}, \tau_{1}$ saturates $P_{2} \backslash P_{1}$ and $\left.\left.\tau_{1}\right|_{Q \backslash P_{2}} \subseteq \tau_{2}\right|_{Q \backslash P_{2}}$.

Lemma 2.4. Let $v=\mathscr{C}(\sigma, P, \tau)$. Then

$$
\operatorname{ker} v=\operatorname{ker} \sigma \cup\left\{a \in P^{*} \mid a^{\prime} \in \operatorname{ker} \sigma \text { for some } a^{\prime} \in S \sigma \text {-linked to } a\right\} \cup(\operatorname{ker} \tau)^{*} \text {. }
$$

Proof. Let $a \in V$. For $a \in S$, clearly $a \in \operatorname{ker} v$ if and only if $a \in \operatorname{ker} \sigma$. Similarly, for $a \in Q \backslash P$, clearly $a \in \operatorname{ker} v$ if and only if $a \in \operatorname{ker} \tau$. 
Next let $a \in P^{*}$. Assume first that $a \in \operatorname{ker} v$. Then ave for some $e \in E(V)$ and we must have $e \in E\left(S \cup P^{*}\right)$. Let $a^{\prime}$ be an element of $S \sigma$-linked to $a$. Then $a^{\prime} v e$ and $a^{\prime} v \cap S$ is an idempotent $\sigma$-class and thus must contain an idempotent, say $f$. Then $a^{\prime} \sigma f$ so that $a^{\prime} \in \operatorname{ker} \sigma$. Conversely, suppose that $a^{\prime} \in \operatorname{ker} \sigma$ for some $a^{\prime} \in S \sigma$-linked to $a$. Then $a^{\prime} \sigma e$ for some $e \in E(S)$ and thus ava've so that $a \in \operatorname{ker} v$.

A mapping $\varphi: Q^{*} \rightarrow S$ is a partial homomorphism if for any $a, b \in Q^{*}, a b \neq 0$ in $Q$ implies $(a b) \varphi=(a \varphi)(b \varphi)$. If in addition,

$$
a b=\left\{\begin{array}{ccc}
(a \varphi) b & \text { if } & a \in Q^{*}, b \in S, \\
a(b \varphi) & \text { if } & a \in S, b \in Q^{*}, \\
(a \varphi)(b \varphi) & \text { if } & a, b \in Q^{*}, a b \in S,
\end{array}\right.
$$

then the multiplication in $V$ is determined by $\varphi$ and $V$ is a strict extension of $S$.

Starting with Section 4, we assume that $V$ is a strict extension of $S$, where the multiplication is determined by the partial homomorphism $\varphi: Q^{*} \rightarrow S$. The mapping $\psi=\varphi \cup \iota_{S}$ is a retraction of $V$ onto $S$, where $\iota_{S}$ is the identity mapping on $S$. If $1 \in V^{1}$ and $1 \notin V$, we write $1 \varphi=1 \psi=1 \in S^{1}$.

In such a case, we have the following important simplification.

Lemma 2.5. ([3, Proposition 2]). Let $V$ be a strict extension of $S$, where the multiplication is determined by a partial homomorphism $\varphi: Q^{*} \rightarrow S$. Let $\sigma \in \mathscr{C}(S), P$ be an ideal of $Q$ and $\tau \in \mathscr{C}_{0}(Q / P)$. Then $(\sigma, P, \tau)$ is an admissible triple if and only if

$$
a, b \in Q \backslash P, a \tau b \Rightarrow a \varphi \sigma b \varphi \text {. }
$$

Lemma 2.6. Let $V$ be a strict extension of $S$, where the multiplication is determined by a partial homomorphism $\varphi: Q^{*} \rightarrow S$. Let $v=\mathscr{C}(\sigma, P, \tau)$. Then

$$
\operatorname{ker} v=\operatorname{ker} \sigma \cup\left\{a \in P^{*} \mid a \varphi \in \operatorname{ker} \sigma\right\} \cup(\operatorname{ker} \tau)^{*} .
$$

Proof. This is a direct consequence of Lemma 2.4.

3. The trace relation. A technical lemma is needed here in order to characterize the relation $T$ on $\mathscr{C}(V)$ in terms of $T$ on $\mathscr{C}(S)$ and $\mathscr{C}(Q / P)$, where $P$ is an ideal of $Q$.

Lemma 3.1. Let $v_{i}=\mathscr{C}\left(\sigma_{i}, Q, \epsilon\right)$ for $i=1,2, \sigma_{1} T \sigma_{2}$, and $e \in E\left(Q^{*}\right)$. Then there exist $e_{1}, e_{2} \in E(S)$ such that $e_{1} v_{1} e v_{2} e_{2}$ and $e_{1} \sigma_{1} \wedge \sigma_{2} e_{2}$.

Proof. Let $i=1,2$. Since $e v_{i} \cap S$ is an idempotent $\sigma_{i}$-class, it contains an idempotent, $e_{i}$ say. Clearly $e_{1} v_{1} e v_{2} e_{2}$; we shall show that $e_{1} \sigma_{1} \wedge \sigma_{2} e_{2}$. First let $u_{i}$ be an inverse of $e e_{i}$ and let $g_{i}=e e_{i} u_{i} e$. Then

$$
\begin{gathered}
g_{i}^{2}=\left(e e_{i} u_{i} e e_{i}\right) u_{i} e=e e_{i} u_{i} e=g_{i} \in E(S), \\
g_{i}=e e_{i} u_{i} e v_{i} e e_{i} u_{i} e e_{i}=e e_{i} v_{i} e_{i},
\end{gathered}
$$

so that $g_{i}<e$ and $g_{i} v_{i} e, i=1,2$. Here $<$ is the natural partial order on the idempotents. Now $e v_{2} g_{2}$ implies $g_{1}=g_{1} e v_{2} g_{1} g_{2}$ and $g_{1}=e g_{1} v_{2} g_{2} g_{1}$ so that

$$
g_{1} \sigma_{2} g_{1} g_{2} \sigma_{2} g_{2} g_{1}
$$

Interchanging the roles of $g_{1}, g_{2}$ and $\sigma_{1}, \sigma_{2}$, we obtain

$$
g_{2} \sigma_{1} g_{2} g_{1} \sigma_{1} g_{1} g_{2}
$$


Let $v$ be an inverse of $g_{1} g_{2}$ and let $h=g_{1} g_{2} v g_{1}$. Then $h \in E(S)$ and we get

$$
h=g_{1} g_{2} v g_{1} \sigma_{2} g_{1} g_{2} v g_{1} g_{2}=g_{1} g_{2} \sigma_{2} g_{1} \text {. }
$$

Hence $g_{1} \sigma_{2} h$ and the hypothesis implies that $g_{1} \sigma_{1} h$. Together with (2), this gives $g_{1} \sigma_{1} g_{1} g_{2} v g_{1} \sigma_{1} g_{2} v g_{1}$ whence

$$
g_{1} \sigma_{1} g_{2} g_{1} \text {. }
$$

Similarly, we let $w$ be an inverse $g_{2} g_{1}$ and $t=g_{1} w g_{2} g_{1}$. Then $t \in E(S)$ and by (1), we obtain

$$
t=g_{1} w g_{2} g_{1} \sigma_{2} g_{2} g_{1} w g_{2} g_{1}=g_{2} g_{1} \sigma_{2} g_{1} \text {. }
$$

Hence $g_{1} \sigma_{2} t$ and the hypothesis implies that $g_{1} \sigma_{1} t$. Together with (2), this gives $g_{1} \sigma_{1} g_{1} w g_{2} g_{1} \sigma_{1} g_{1} w g_{2}$ whence $g_{1} \sigma_{1} g_{1} g_{2}$. This together with (1) and (3) yields

$$
g_{1} \sigma_{1} \wedge \sigma_{2} g_{1} g_{2} \sigma_{1} \wedge \sigma_{2} g_{2} g_{1}
$$

Now interchanging the roles of $g_{1}, g_{2}$ and $\sigma_{1}, \sigma_{2}$ we obtain

$$
g_{2} \sigma_{1} \wedge \sigma_{2} g_{2} g_{1} \sigma_{1} \wedge \sigma_{2} g_{1} g_{2}
$$

which together with (4) yields

$$
g_{1} \sigma_{1} \wedge \sigma_{2} g_{2}
$$

We have seen above that $e_{i} v_{i} e v_{i} g_{i}$ so that $e_{i} \sigma_{i} g_{i}$ for $i=1,2$. The hypothesis implies that $e_{1} \sigma_{1} g_{1}$ gives $e_{1} \sigma_{2} g_{1}$ and $e_{2} \sigma_{2} g_{2}$ gives $e_{2} \sigma_{1} g_{2}$. This together with (5) finally yields

$$
e_{1} \sigma_{1} \wedge \sigma_{2} g_{1} \sigma_{1} \wedge \sigma_{2} g_{2} \sigma_{1}^{\prime} \wedge \sigma_{2} e_{2}
$$

so that $e_{1} \sigma_{1} \wedge \sigma_{2} e_{2}$, as required.

We are now ready for the relation $T$.

TheOrem 3.2. Let $v_{i}=\mathscr{C}\left(\sigma_{i}, P_{i}, \tau_{i}\right)$ for $i=1,2$. Then

$$
v_{1} T v_{2} \Leftrightarrow \sigma_{1} T \sigma_{2}, \quad P_{1}=P_{2}, \quad \tau_{1} T \tau_{2} .
$$

Proof. (a) $\Rightarrow$. If $e, f \in E(S)$ are such that $e \sigma_{1} f$, then $e v_{1} f$ so by hypothesis $e v_{2} f$ whence $e \sigma_{2} f$. Therefore tr $\sigma_{1} \subseteq \operatorname{tr} \sigma_{2}$ and by symmetry, $\sigma_{1} T \sigma_{2}$.

Let $e \in E\left(P_{1} \backslash P_{2}\right)$. Then $e v_{1} \cap S$ is an idempotent $\sigma_{1}$-class so it contains an idempotent, $f$ say. Hence $e v_{1} f$ which by hypothesis implies that $e v_{2} f$ which is impossible since $e \notin P_{2}$ and $v_{2}$ saturates $S \cup P_{2}^{*}$. Therefore $E\left(P_{1} \backslash P_{2}\right)=\varnothing$. If $a \in P_{1} \backslash P_{2}$, then for any inverse $a^{\prime}$ of $a$, we have $a a^{\prime} \in P_{1} \backslash P_{2}$ which we have just seen to be impossible. Thus $P_{1} \backslash P_{2}=\varnothing$ that is $P_{1} \subseteq P_{2}$. The equality $P_{1}=P_{2}$ now follows by symmetry.

If $e, f \in E(T \backslash P)$ are such that $e \tau_{1} f$, then $e v_{1} f$ so by hypothesis $e v_{2} f$ whence $e \tau_{2} f$. Therefore tr $\tau_{1} \subseteq \operatorname{tr} \tau_{2}$ and by symmetry, $\tau_{1} T \tau_{2}$.

(b) $\Leftarrow$. Let $e, f \in E(V)$ be such that $e v_{1} f$. If $e, f \in S$, then $\sigma_{1} T \sigma_{2}$ implies that $e v_{2} f$, and if $e, f \in Q \backslash P$, then $\tau_{1} T \tau_{2}$ implies that $e v_{2} f$. Consider the case $e, f \in P_{1}^{*}$. By Lemma 3.1 , there exist $e_{1}, e_{2}, f_{1}, f_{2} \in E(S)$ such that

$$
\left.\begin{array}{lc}
e_{1} v_{1} e v_{2} e_{2}, & f_{1} v_{1} f v_{2} f_{2}, \quad e v_{1} f \\
e_{1} \sigma_{1} \wedge \sigma_{2} e_{2}, & f_{1} \sigma_{1} \wedge \sigma_{2} f_{2} .
\end{array}\right\}
$$


Consequently $e_{1} v_{1} e v_{1} f v_{1} f_{1}$ so that $e_{1} \sigma_{1} f_{1}$ which by hypothesis gives $e_{1} \sigma_{2} f$ whence

$$
e v_{2} e_{2} \sigma_{2} e_{1} \sigma_{2} f_{1} \sigma_{2} f_{2} v_{2} f
$$

and thus $e v_{2} f$.

By symmetry, it remains to consider the case $e \in P_{1}^{*}, f \in S$. With the above notation, we have $e_{1} v_{1} e v_{1} f$ so that $e_{1} \sigma_{1} f$ which by hypothesis gives $e_{1} \sigma_{2} f$ where $e v_{2} e_{2} \sigma_{2} e_{1} f$ and thus $e v_{2} f$.

Therefore in all cases $e v_{2} f$ which proves that $\operatorname{tr} v_{1} \subseteq \operatorname{tr} v_{2}$ and by symmetry equality prevails. Consequently $v_{1} T v_{2}$.

It would be natural to attempt to characterize $v_{T}$ and $v^{T}$ in terms of admissible triples when $v$ itself is given in this form. In this generality, this does not seem feasible. We limit ourselves to the following special case.

Corollary 3.3. Let $v=\mathscr{C}(\sigma, Q, \epsilon)$. Then $v^{T}=\left(\sigma^{T}, Q, \epsilon\right)$.

Proof. Since $(\sigma, Q, \epsilon)$ is an admissible triple, so is $\left(\sigma^{T}, Q, \epsilon\right)$. Let $\theta=\mathscr{C}\left(\sigma^{T}, Q, \epsilon\right)$. By Theorem 3.2, we have $v T \theta$. Next let $v^{\prime}=\mathscr{C}\left(\sigma^{\prime}, P^{\prime}, \tau^{\prime}\right)$ be such that $v^{\prime} T v$. By Theorem 3.2, we get $\sigma^{\prime} T \sigma, P=Q$ and $\tau^{\prime}=\epsilon$. Hence $\sigma^{\prime} \subseteq \sigma^{T}$ which by Lemma 2.3 implies that $v^{\prime} \subseteq \theta$. This proves the required maximality of $\theta$.

A similar analysis for the kernel relation is not possible because of the fact that a situation of the form $\mathscr{C}(\sigma, P, \tau) K \mathscr{C}\left(\sigma^{\prime}, P^{\prime}, \tau^{\prime}\right)$ with $P \neq P^{\prime}$ is possible. We limit ourselves only to the analogue of Corollary 3.3 for the kernel.

Proposition 3.4. Let $v=\mathscr{C}(\sigma, Q, \epsilon)$. Then $v^{\kappa}=\mathscr{C}\left(\sigma^{\kappa}, Q, \epsilon\right)$.

Proof. Since $(\sigma, Q, \epsilon)$ is an admissible triple so is $\left(\sigma^{K}, Q, \epsilon\right)$. Let $\theta=\mathscr{C}\left(\sigma^{K}, Q, \epsilon\right)$. By Lemma 2.4, we have

$$
\begin{gathered}
\operatorname{ker} v=\operatorname{ker} \sigma \cup\left\{a \in Q^{*} \mid a^{\prime} \in \operatorname{ker} \sigma \text { for some } a^{\prime} \in S \sigma \text {-linked to } a\right\}, \\
\operatorname{ker} \theta=\operatorname{ker} \sigma^{\kappa} \cup\left\{a \in Q^{*} \mid a^{\prime \prime} \in \operatorname{ker} \sigma^{K} \text { for some } a^{\prime \prime} \in S \sigma^{K} \text {-linked to } a\right\},
\end{gathered}
$$

where $\operatorname{ker} \sigma=\operatorname{ker} \sigma^{K}$. If $a \in Q^{*}$ and $a^{\prime} \in \operatorname{ker} \sigma$ are $\sigma$-linked, they are also $\sigma^{K}$-linked so that (7) is contained in (8). Conversely, let $a \in Q^{*}$ and $a^{\prime \prime} \in \operatorname{ker} \sigma$ be $\sigma^{\kappa}$-linked. Then for all $x \in S$, we have $a x \sigma^{K} a^{\prime \prime} x$ and $x a \sigma^{K} x a^{\prime \prime}$. Now let $a^{\prime} \in S$ be such that $a, a^{\prime}$ are $\sigma$-linked. Then for all $x \in S$, we have $a x \sigma a^{\prime} x$ and $x a \sigma x a^{\prime}$ which implies that also $a x \sigma^{\kappa} a^{\prime} x$ and $x a \sigma^{K} x a^{\prime}$. It follows that $a^{\prime} x \sigma^{K} a^{\prime \prime} x$ and $x a^{\prime} \sigma^{K} x a^{\prime \prime}$ for all $x \in S$. Recall that a semigroup $T$ is weakly reductive if for any $a, b \in T, x a=x b$ and $a x=b x$ for all $x \in T$ implies that $a=b$. Weak reductivity of $S / \sigma^{K}$ gives that $a^{\prime} \sigma^{K} a^{\prime \prime}$. Since $a^{\prime \prime} \in \operatorname{ker} \sigma^{K}$, we must have that $a^{\prime} \in \operatorname{ker} \sigma^{K}=\operatorname{ker} \sigma$. Therefore (8) is contained in (7) and equality prevails so that $v K \theta$.

Next let $v^{\prime}=\mathscr{C}\left(\sigma^{\prime}, P^{\prime}, \tau^{\prime}\right)$ be such that $v^{\prime} K v$. By Lemma 2.4 we have an expression for $\operatorname{ker} v^{\prime}$ analogous to that for ker $v$ in (7) which implies that $\operatorname{ker} \sigma^{\prime}=\operatorname{ker} \sigma$. It follows that $\sigma^{\prime} K \sigma$ and thus $\sigma^{\prime} \subseteq \sigma^{K}$. The remaining three conditions in Lemma 2.3 are trivially satisfied which gives that $v^{\prime} \subseteq \theta$ which establishes the required maximality of $\theta$. 
4. The lower and upper ends of trace classes. We reiterate first that henceforth $V$ is a strict extension of $S$, where the multiplication is determined by a partial homomorphism $\varphi: Q^{*} \rightarrow S$. This will not be stated explicitly. Besides characterizing the ends, we include several consequences of these results.

TheOrem 4.1. Let $v=\mathscr{C}(\sigma, P, \tau)$. Then $v_{T}=\mathscr{C}\left(\sigma_{T}, P, \tau_{T}\right)$.

Proof. In view of Theorem 3.2, it suffices to show that $\left(\sigma_{T}, P, \tau_{T}\right)$ is an admissible triple.

Let $\eta=\operatorname{tr} \sigma$ and $\theta=\operatorname{tr} \tau$. By Lemma 2.2, we have $\sigma_{T}=\eta^{*}$ and $\tau_{T}=\theta^{*}$, the first of these taken within $S$ and the second one within $Q / P$. Let $a \tau_{T} b$ for $a, b \in Q \backslash P$. Then there is a sequence

$$
a=x_{1} e_{1} y_{1}, \quad x_{1} f_{1} y_{1}=x_{2} e_{2} y_{2}, \ldots, x_{n} f_{n} y_{n}=b
$$

for some $x_{i}, y_{i} \in(Q / P)^{1}$ and $e_{i} \theta f_{i}, i=1,2, \ldots, n$. Since $\tau$ is 0 -restricted and $\tau_{r} \subseteq \tau$, we have that $\tau_{T}$ is 0 -restricted. In the above sequence, we have

$$
a \tau_{T} x_{1} f_{1} y_{1} \tau_{T} x_{2} f_{2} y_{2} \ldots \tau_{T} b
$$

which together with $a \neq 0$ in $Q / P$ implies that all these elements are nonzero in $Q / P$. We may thus apply $\varphi$ to the sequence $(9)$ thereby obtaining

$$
a \varphi=\left(x_{1} \varphi\right)\left(e_{1} \varphi\right)\left(y_{1} \varphi\right),\left(x_{1} \varphi\right)\left(f_{1} \varphi\right)\left(y_{1} \varphi\right)=\left(x_{2} \varphi\right)\left(e_{2} \varphi\right)\left(y_{2} \varphi\right) \ldots\left(x_{n} \varphi\right)\left(f_{n} \varphi\right)\left(y_{n} \varphi\right)=b \varphi
$$

with $x_{i} \varphi, y_{i} \varphi \in S^{\prime}$. In addition $e_{i} \tau f_{i}$ implies that $e_{i} \varphi \sigma f_{i} \varphi$ by Lemma 2.5 so that $e_{i} \varphi \eta f_{i} \varphi$. Since $\sigma_{T}=\eta^{*}$ by Lemma 2.2, we get $a \varphi \sigma_{T} b \varphi$. By Lemma $2.5,\left(\sigma_{T}, P, \tau_{T}\right)$ is an admissible triple and therefore $v_{T}=\mathscr{C}\left(\sigma_{T}, P, \tau_{T}\right)$.

THEOREM 4.2. Let $v=\mathscr{C}(\sigma, P, \tau)$. Then $v^{T}=\mathscr{C}\left(\sigma^{T}, P, \tau^{T} \cap \bar{\sigma}\right)$ where $a \bar{\sigma} b$ if $a, b \in Q \backslash P, \quad a \varphi \sigma^{T} b \varphi, \quad 0 \bar{\sigma} 0$.

Proof. Let $v^{T}=\mathscr{C}\left(\sigma^{\prime}\right.$, , ) where the blanks stand for entries to be determined. By Theorem 3.2, $\sigma T \sigma^{\prime}$ and thus $\sigma^{\prime} \subseteq \sigma^{T}$. Let $a \sigma^{T} b$. Then for $\theta=\operatorname{tr} \sigma$, by Lemma 2.2, we have

$$
a(\mathscr{L} \theta \mathscr{L} \theta \mathscr{L} \cap \mathscr{R} \theta \mathscr{R} \theta \mathscr{R})^{10} b
$$

within $S$ and thus for every $x, y \in S^{1}$,

$$
\text { xay } \mathscr{L} \theta \mathscr{L} \theta \mathscr{L} \cap \mathscr{R} \theta \mathscr{R} \theta \mathscr{R} x b y .
$$

Let $\theta^{\prime}=\operatorname{tr} v$. Then $\theta=\left.\theta^{\prime}\right|_{s}$ and for any $x, y \in V^{\prime}$,

$$
(x \psi) a(y \psi) \mathscr{L} \theta^{\prime} \mathscr{L} \theta^{\prime} \mathscr{L} \cap \mathscr{R} \theta^{\prime} \mathscr{R} \theta^{\prime} \mathscr{R}(x \psi) b(y \psi)
$$

and thus, for any $x, y \in V^{\prime}$,

$$
x a y \mathscr{L} \theta^{\prime} \mathscr{L} \theta^{\prime} \mathscr{L} \cap \mathscr{R} \theta^{\prime} \mathscr{R} \theta^{\prime} \mathscr{R} x b y
$$

so that $a\left(\mathscr{L} \theta^{\prime} \mathscr{L} \theta^{\prime} \mathscr{L} \cap \mathscr{R} \theta^{\prime} \mathscr{R} \theta^{\prime} \mathscr{R}\right)^{\prime} b$ within $V$. Therefore $a v^{\top} b$ whence $a \sigma^{\prime} b$. Consequently $\sigma^{T} \subseteq \sigma^{\prime}$ and equality prevails. From Theorem 3.2 we get that $v^{T}=$ $\mathscr{C}\left(\sigma^{T}, P,\right)$ where the blank stands for the entry to be determined. 
Clearly $\bar{\sigma}$ is an equivalence relation on $Q / P$. The set $0 \tau^{T}$ is an ideal of $Q$. If it contains a nonzero element, it also contains a nonzero idempotent, say $e$. But then $e \tau^{T} 0$ so that $e \tau 0$, which contradicts the hypothesis that $\tau$ is 0 -restricted.

Hence also $\tau^{T}$ is 0 -restricted. Set $\eta=\tau^{T} \cap \bar{\sigma}$. Next let $a, b \in Q \backslash P$ be such that $a \eta b$ and let $c \in Q \backslash P$. If $a c \neq 0$ in $Q / P$, then $a c \tau^{T} b c$ implies $b c \neq 0$ and $a \bar{\sigma} b$ gives $(a c) \varphi=$ $(a \varphi)(c \varphi) \sigma^{T}(b \varphi)(c \varphi)=(b c) \varphi$ so that $a c \bar{\sigma} b c$. If $a c=0$ in $Q / P$, then $a c \tau^{T} b c$ implies $b c=0$. Therefore $a c \eta b c$ in all cases; similarly $c a \eta c b$ which proves that $\eta$ is a congruence on $Q / P$. Trivially $\eta$ is 0 -restricted. By Lemma 2.5 , we conclude that $\left(\sigma^{T}, P, \eta\right)$ is an admissible triple; let $\xi=\mathscr{C}\left(\sigma^{T}, P, \eta\right)$.

If $e, f \in E(Q \backslash P)$ are such that $e \tau f$, then by Lemma 2.5 we have $e \varphi \sigma f \varphi$ whence $e \varphi \sigma^{T} f \varphi$. It follows that $\operatorname{tr} \tau \subseteq \operatorname{tr}\left(\tau^{T} \cap \bar{\sigma}\right)=\operatorname{tr} \eta$. Conversely, trivially $\operatorname{tr} \eta \subseteq \operatorname{tr} \tau^{T}=\operatorname{tr} \tau$ and thus $\operatorname{tr} \tau=\operatorname{tr} \eta$. Therefore $\tau T \eta$ which by Theorem 3.2 implies that $v T \xi$.

Finally, let $v^{\prime}=\mathscr{C}\left(\sigma^{\prime}, P^{\prime}, \tau^{\prime}\right)$ be such that $v^{\prime} T v$. By Theorem 3.2, we get $\sigma^{\prime} T \sigma$, $P^{\prime}=P$ and $\tau^{\prime} T \tau$. Hence $\sigma^{\prime} \subseteq \sigma^{T}$ and $\tau^{\prime} \subseteq \tau^{T}$. If $a, b \in Q \backslash P$ are such that $a \tau^{\prime} b$, then by Lemma 2.5 we have $a \varphi \sigma^{\prime} b \varphi$ whence $a \varphi \sigma^{T} b \varphi$ so that $a \bar{\sigma} b$. Therefore $\tau^{\prime} \subseteq \bar{\sigma}$ which implies that $\tau^{\prime} \subseteq \eta$. Now Lemma 2.3 implies that $v^{\prime} \subseteq \xi$ which establishes the desired maximality of $\xi$.

In the next consequence of the above theorem we have a case in which $\bar{\sigma}$ in the theorem may be omitted.

Corollary 4.3. Let $v=\mathscr{C}(\sigma, P, \tau)$ and assume that $\varphi$ maps $Q \backslash P$ onto $S$. Then $v^{T}=\mathscr{C}\left(\sigma^{T}, P, \tau^{T}\right)$.

Proof. In view of Theorem 4.2, it suffices to prove that $\tau^{T} \subseteq \bar{\sigma}$. Hence let $a \tau^{T} b$, $\theta=\operatorname{tr} \tau$ and $\eta=\operatorname{tr} \sigma$. By Lemma 2.2, for any $x, y \in(Q / P)^{1}$, we have

$$
\text { xay } \mathscr{L} \theta \mathscr{L} \theta \mathscr{L} \cap \mathscr{R} \theta \mathscr{R} \theta \mathscr{R} x \text { by }
$$

so that

$$
\text { xay } \mathscr{L} e \theta f \mathscr{L} g h \mathscr{L} x \text { by }, \quad \text { xay } \mathscr{R} e^{\prime} \theta f^{\prime} \mathscr{R} g^{\prime} \theta h^{\prime} \mathscr{R} x \text { by }
$$

for some $e, f, g, h, e^{\prime}, f^{\prime}, g^{\prime}, h^{\prime} \in E(Q / P)$. Since $\mathscr{L}, \mathscr{R}$ and $\theta$ are 0-restricted, we have $x a y \neq 0$ if and only if $x b y \neq 0$. Now assuming that $x a y \neq 0$, we may apply $\varphi$ to the sequences in (10) so that by Lemma 2.5 , writing $1 \varphi=1$, we obtain

$$
\begin{gathered}
(x \varphi)(a \varphi)(y \varphi) \mathscr{L} e \varphi \eta f \varphi \mathscr{L} g \varphi \eta h \varphi \mathscr{L}(x \varphi)(b \varphi)(y \varphi), \\
(x \varphi)(a \varphi)(y \varphi) \mathscr{R} e^{\prime} \varphi \eta f^{\prime} \varphi \mathscr{R} g^{\prime} \varphi \eta h^{\prime} \varphi \mathscr{R}(x \varphi)(b \varphi)(y \varphi)
\end{gathered}
$$

and thus

$$
(x \varphi)(a \varphi)(y \varphi) \mathscr{L} \eta \mathscr{L} \eta \mathscr{L} \cap \mathscr{R} \eta \mathscr{R} \eta \mathscr{R}(x \varphi)(b \varphi)(y \varphi) .
$$

Since this holds for all $x, y \in(Q \backslash P) \cup\{1\}$ and $\varphi$ maps $Q \backslash P$ onto $S$, we conclude that $a \varphi(\mathscr{L} \eta \mathscr{L} \eta \mathscr{L} \cap \mathscr{R} \eta \mathscr{R} \eta \mathscr{R})^{\prime \prime} b \varphi$ so that by Lemma $2.2 a \varphi \sigma^{T} b \varphi$ and thus $a \bar{\sigma} b$.

The next result provides a copy of the trace class of a congruence on $V$ expressed by means of an admissible triple.

Corollary 4.4. Denote by $\mathscr{A} \mathscr{T}$ the set of all admissible triples and, for $(\sigma, P, \tau) \in$ $\mathscr{A T}$, let

$$
(\sigma, P, \tau) T=\left\{\left(\sigma^{\prime}, P^{\prime}, \tau^{\prime}\right) \in \mathscr{A} \mathscr{T} \mid \mathscr{C}\left(\sigma^{\prime}, P^{\prime}, \tau^{\prime}\right) T \mathscr{C}(\sigma, P, \tau)\right\}
$$


Then, for any $(\sigma, P, \tau) \in \mathscr{A} \mathscr{T}$, we have

$$
(\sigma, P, \tau) T=\left(\left[\sigma_{T}, \sigma^{T}\right] \times\{P\} \times\left[\tau_{T}, \tau^{T} \cap \bar{\sigma}\right]\right) \cap \mathscr{A} \mathscr{T} .
$$

Proof. Let $\left(\sigma^{\prime}, P^{\prime}, \tau^{\prime}\right) \in(\sigma, P, \tau) T$. Then

$$
(\mathscr{C}(\sigma, P, \tau))_{T} \subseteq \mathscr{C}\left(\sigma^{\prime}, P^{\prime}, \tau^{\prime}\right) \subseteq(\mathscr{C}(\sigma, P, \tau))^{T}
$$

which by Theorems 4.1 and 4.2 gives

$$
\mathscr{C}\left(\sigma_{T}, P, \tau_{T}\right) \subseteq \mathscr{C}\left(\sigma^{\prime}, P^{\prime}, \tau^{\prime}\right) \subseteq \mathscr{C}\left(\sigma^{T}, P, \tau^{T} \cap \bar{\sigma}\right)
$$

which in turn implies that $\sigma_{T} \subseteq \sigma^{\prime} \subseteq \sigma^{T}, P=P^{\prime}$ and $\tau_{T} \subseteq \tau^{\prime} \subseteq \tau^{T} \cap \bar{\sigma}$. It follows that $\left(\sigma^{\prime}, P^{\prime}, \tau^{\prime}\right)$ is contained in the right hand side of (11). The proof of the converse follows essentially by reversing the steps above.

5. The lower and upper ends of kernel classes. We continue with the hypothesis that $V$ is a strict extension of $S$ and characterize these ends including some special cases.

THEOREM 5.1. Let $v=\mathscr{C}(\sigma, P, \tau)$. Then $v_{K}=\mathscr{C}\left(\sigma_{K}, P^{\prime}, \tau^{\prime}\right)$ where

$$
A=\left\{a \in P^{*} \mid a \varphi \in \operatorname{ker} \sigma\right\} \cup(\operatorname{ker} \tau)^{*}
$$

and $P^{\prime}$ is the set of all $a$ in $Q^{*}$ for which there exists a sequence

$$
a=x_{1} u_{1} y_{1}, x_{1} v_{1} y_{1}=x_{2} u_{2} y_{2}, \ldots, x_{n-1} v_{n-1} y_{n-1}=x_{n} u_{n} y_{n}
$$

in $Q^{*}, x_{n} v_{n} y_{n} \in S$ with $x_{i}, y_{i} \in\left(Q^{1}\right)^{*}$ and

$$
\left\{u_{i}, v_{i}\right\}=\left\{z_{i}, z_{i}^{2}\right\}, \quad z_{i} \in A \text { for } i=1,2, \ldots, n-1,
$$

and for $i=n$, either the same condition or $v_{n}=u_{n}^{2}, u_{n} \in A, B=\left[A \cap\left(Q \backslash P^{\prime}\right)\right] \cup\{0\}$ and $\tau^{\prime}=\left(\pi_{B}^{Q^{\prime \prime}}\right)_{K}$.

Proof. Let $v_{K}=\mathscr{C}(\xi, R, \eta)$. By Lemma 2.6, we have

$$
\begin{aligned}
& \operatorname{ker} v=\operatorname{ker} \sigma \cup\left\{a \in P^{*} \mid a \varphi \in \operatorname{ker} \sigma\right\} \cup(\operatorname{ker} \tau)^{*}, \\
& \operatorname{ker} v_{K}=\operatorname{ker} \xi \cup\left\{a \in R^{*} \mid a \varphi \in \operatorname{ker} \xi\right\} \cup(\operatorname{ker} \eta)^{*}
\end{aligned}
$$

and $v K v_{K}$ implies that ker $\sigma=\operatorname{ker} \xi$ and

$$
A=\left\{a \in R^{*} \mid a \varphi \in \operatorname{ker} \sigma\right\} \cup(\operatorname{ker} \eta)^{*}=\operatorname{ker} v_{K} \cap Q^{*} .
$$

The former implies that $\sigma K \xi$ whence $\sigma_{K} \subseteq \xi$.

Next let $a \xi b$. Then $a v_{K} b$ and thus there exists a sequence of the form

$$
\left.\begin{array}{l}
a=x_{1} u_{1} y_{1}, x_{1} v_{1} y_{1}=x_{2} u_{2} v_{2}, \ldots, x_{n} v_{n} y_{n}=b \text { for some } \\
x_{i}, y_{i} \in V^{1},\left\{u_{i}, v_{i}\right\}=\left\{z_{i}, z_{i}^{2}\right\}, z_{i} \in \operatorname{ker} v \text { for } i=1,2, \ldots, n .
\end{array}\right\}
$$

Then (16) implies that

$$
a=\left(x_{1} \psi\right)\left(u_{1} \psi\right)\left(y_{1} \psi\right),\left(x_{1} \psi\right)\left(v_{1} \psi\right)\left(y_{1} \psi\right)=\left(x_{2} \psi\right)\left(u_{2} \psi\right)\left(v_{2} \psi\right), \ldots,\left(x_{n} \psi\right)\left(v_{n} \psi\right)\left(y_{n} k\right)=b
$$

with $\left\{u_{i} \psi, v_{i} \psi\right\}=\left\{z_{i} \psi,\left(z_{i} \psi\right)^{2}\right\}$, and in view of (14), $z_{i} \psi \in \operatorname{ker} \sigma$. Therefore $a \sigma_{K} b$ which implies that $\xi \subseteq \sigma_{K}$ and equality prevails.

Note that

$$
R=\left\{a \in Q^{*} \mid a v_{K} b \text { for some } b \in S\right\} \cup\{0\} \text {. }
$$


First let $a \in P^{\prime}$ with the notation as in the statement of the theorem. For $i=1,2, \ldots, n$, in view of (12) and (14), $z_{i} \in A$ implies that $z_{i} \in \operatorname{ker} v$ and $u_{n} \in A$ implies that $u_{n} \in \operatorname{ker} v$. Hence $a v_{K} x_{n} v_{n} y_{n} \in S$ and thus (17) implies that $a \in R$. Therefore $P^{\prime} \subseteq R$.

Conversely, let $a \in R^{*}$. In view of (17), there exists a sequence of the form (16) with $b \in S$. Since $a=x_{1} u_{1} y_{1} \notin S$, there exists a least positive integer $j$ such that $x_{i} u_{i} y_{i} \notin S$ for all $i \leqslant j$. Without loss of generality, we may assume that $j=n-1$. We thus have arrived at a sequence in $Q^{*}$ of the form (13). It follows that $x_{i}, y_{i} \in\left(Q^{1}\right)^{*}$ for $i=1,2, \ldots, n$. In view of (15), we also have $z_{i} \in A$ for $i=1,2, \ldots, n-1$. For $i=n$, we have $\left\{u_{n}, v_{n}\right\}=\left\{z_{n}, z_{n}^{2}\right\}$ with $z_{n} \in \operatorname{ker} v$ and also $x_{n} u_{n} y_{n} \notin S, x_{n} v_{n} y_{n} \in S$. If $z_{n}^{2} \in Q^{*}$, then $z_{n} \in Q^{*}$ and thus $z_{n} \in A$. Otherwise $z_{n}^{2} \in S$ and we must have $v_{n}=z_{n}^{2}$ so that $u_{n}=z_{n}$ whence $v_{n}=u_{n}^{2}$ and $u_{n} \in A$. Therefore $a \in P^{\prime}$. Consequently $R \subseteq P^{\prime}$ and equality prevails.

Now let $a, b \in Q \backslash P^{\prime}$. Assume first that $a \eta b$. Then $a v b$ and we have a sequence of the form (16). Here $z_{i} \in \operatorname{ker} v$ and since $a, b \in Q \backslash P^{\prime}$, we must have, in view of (15), that

$$
z_{i} \in \operatorname{ker} v \cap\left(Q \backslash P^{\prime}\right)=A \cap\left(Q \backslash P^{\prime}\right)=B^{*} .
$$

In particular, $B=\operatorname{ker} \eta$ which in view of $\left(\left[2\right.\right.$, Theorem 2.13]) yields that $B=\operatorname{ker} \pi_{B}{ }^{\prime P^{\prime}}$. Now the sequence (16) where $z_{i} \in \operatorname{ker} \pi_{B}^{Q^{\prime P^{\prime}}}$ gives that $a\left(\pi_{B}^{Q^{\prime P^{\prime}}}\right)_{K} b$, that is $a \tau^{\prime} b$. Since $\eta$ is 0 -restricted, this shows that $\eta \subseteq \tau^{\prime}$. Furthermore, $\operatorname{ker} \eta=B=\operatorname{ker} \pi_{B}^{Q / P^{\prime}}=\operatorname{ker} \tau^{\prime}$ so that $\tau^{\prime}=\tau_{K}^{\prime} \subseteq \eta$ and equality prevails.

Corollary 5.2. Let $v=\mathscr{C}(\sigma,\{0\}, \tau)$. Then $v_{K}=\mathscr{C}\left(\sigma_{K},\{0\}, \tau_{K}\right)$.

Proof. For $P=\{0\}$ in Theorem 5.1, we have $A=(\operatorname{ker} \tau)^{*}$ and $P^{\prime}=\{0\}$ so that $B=\operatorname{ker} \tau$ and thus $\tau^{\prime}=\left(\pi_{\text {ker } \tau}\right)_{K}=\left(\tau^{K}\right)_{K}=\tau_{K}$.

Corollary 5.3. Assume that $Q$ is categorical at zero. Let $v=\mathscr{C}(\sigma, P, \tau)$ and suppose that $a \in P^{*}$ and $a \varphi \in \operatorname{ker} \sigma$ imply that $a^{2} \in Q^{*}$. Then in the notation of Theorem 5.1, $P^{\prime}=\{0\}$ and $B=A \cup\{0\}$.

Proof. We adopt the notation of Theorem 5.1. Let $a \in P^{\prime *}$ and write $x_{n}, u_{n}, v_{n}, y_{n}$, $z_{n}$ without subscripts. Then $x u y \neq 0$ in $Q$. Also $\{u, v\}=\left\{z, z^{2}\right\}$ with $z \in A \subseteq Q^{*}$. If $z \in(\operatorname{ker} \tau)^{*}$, then $z^{2} \tau z$ so that $z^{2} \in Q \backslash P$. Assume that $z \in P^{*}$. Then $z \varphi \in \operatorname{ker} \sigma$ and the hypothesis implis that $z^{2} \in Q^{*}$. If $u=z$, then $v=u^{2}$ and thus $x u \neq 0, u u \neq 0$ and $u y \neq 0$ which by categoricity at zero yields $x u^{2} y \neq 0$, that is $x v y \neq 0$. Otherwise $u=z^{2}$ which gives $v^{2}=u$ whence $x v^{2} y \neq 0$ which by categoricity at zero yields $x v y \neq 0$. Therefore $P^{\prime}=\{0\}$. Hence $B=\left(A \cap Q^{*}\right) \cup\{0\}=A \cup\{0\}$.

For a characterization of $v^{K}$ we need a preliminary result. Recall that $J(a)$ denotes the principal ideal generated by $a$.

Lemma 5.4. Let $R \subseteq Q^{*}$. Then

$$
P=\left\{a \in Q^{*} \mid J(a) \cap R=\varnothing\right\} \cup\{0\}
$$

is the union of all ideals $I$ of $Q$ such that $I \cap R=\varnothing$.

Proof. Since $R \subseteq Q^{*}$, we have $\{0\} \cap R=\varnothing$ and hence there exists at least one ideal of $Q$ disjoint from $R$. Let $U$ be the union of all such ideals.

If $a \in P$, then $J(a) \cap R=\varnothing$ and thus $J(a) \subseteq U$ so that $a \in U$. Therefore $P \subseteq U$. Conversely, let $a \in U$. Then there exists an ideal $J$ of $V$ such that $a \in J$ and $J \cap R=\varnothing$. Since $J(a) \subseteq J$, it follows that $J(a) \cap R=\varnothing$ and thus $a \in P$. Therefore $U \subseteq P$ and equality prevails. 
THEOREM 5.5. Let $v=\mathscr{C}(\sigma, P, \tau)$. Then $v^{K}=\mathscr{C}\left(\sigma^{K}, P^{\prime}, \tau^{\prime}\right)$ where

$$
\begin{aligned}
& R=\{a \in Q \backslash \operatorname{ker} \tau \mid a \varphi \in \operatorname{ker} \sigma\}, \\
& P^{\prime}=\left\{a \in Q^{*} \mid J(a) \cap R=\varnothing\right\} \cup P, \\
& a \hat{o} b \text { if } a, b \in Q \backslash P^{\prime}, a \varphi \sigma^{K} b \varphi, 0 \hat{\sigma} 0, \\
& \eta=\left(\left.\tau\right|_{Q \backslash P^{\prime}}\right) \cup\{(0,0)\}, \quad \tau^{\prime}=\eta^{K} \cap \hat{\sigma} \cap \zeta_{Q / P^{\prime}} .
\end{aligned}
$$

Proof. 1. $P^{\prime}$ is an ideal of $Q$ by Lemma 5.4.

2. $\tau$ saturates $P^{\prime} \backslash P$. Indeed, let $a \tau b$ with $a \in Q \backslash P$ and $b \in P^{\prime} \backslash P$. Hence $J(b) \cap R=\varnothing$ and thus, for every $x, y \in Q^{1}, x b y \notin R$. We wish to show that $x a y \notin R$. If $x b y \in \operatorname{ker} \tau$, then $x a y \tau x b y$ implies that $x a y \in \operatorname{ker} \tau$ and thus $x a y \notin R$. Otherwise, $x b y \notin \operatorname{ker} \tau$. Since $x b y \notin R$, we must have $(x b y) \varphi \notin \operatorname{ker} \tau$. The hypothesis $a \tau b$ implies that $a \varphi \sigma b \varphi$ by Lemma 2.5 . Hence $(x a y) \varphi \sigma(x b y) \varphi$ and thus $(x a y) \varphi \notin \operatorname{ker} \sigma$. Also $x b y \notin \operatorname{ker} \tau$ implies that $x a y \notin \operatorname{ker} \tau$. Therefore again $x a y \notin R$. Consequently $a \in P^{\prime}$ and thus $\tau$ saturates $P^{\prime} \backslash P$.

3. $\tau$ saturates $Q \backslash P^{\prime}$ and $\eta \in \mathscr{C}_{0}\left(Q / P^{\prime}\right)$. The first assertion follows from part 2 and the fact that $\tau$ saturates $Q \backslash P$. The second assertion is a consequence of the first.

4. $\tau^{\prime} \in \mathscr{C}_{01}\left(Q / P^{\prime}\right)$. Let $a, b, c \in Q \backslash P^{\prime}$ be such that $a \tau^{\prime} b$ and $a c \in Q \backslash P^{\prime}$. Then $a \zeta_{Q / P^{\prime}} b$ implies that $a c \zeta_{Q / P^{\prime}}, b c$ and thus $b c \neq 0$ in $Q / P^{\prime}$, that is $b c \in Q \backslash P^{\prime}$, since $\zeta_{Q / P^{\prime}}$ is 0 -restricted. In addition $a \eta^{K} b$ implies that $a c \eta^{K} b c$. Finally, $a \varphi \sigma^{\kappa} b \varphi$ implies $(a c) \varphi=$ $(a \varphi)(c \varphi) \sigma^{K}(b \varphi)(c \varphi)=(b c) \varphi$. Therefore $a c \tau^{\prime} b c$. By symmetry, $b c \neq 0$ in $Q / P^{\prime}$ implies $a c \neq 0$ and the same conclusion is reached. Hence $\tau^{\prime}$ is a right congruence and, by duality, it is a congruence. Since $\tau^{\prime} \subseteq \zeta_{Q / P^{\prime}}($ or $\tau \subseteq \hat{\sigma}), \tau^{\prime}$ is 0 -restricted.

5. $\left(\sigma^{\kappa}, P^{\prime}, \tau^{\prime}\right)$ is an admissible triple. Indeed, let $a, b \in Q \backslash P^{\prime}$ be such that $a \tau^{\prime} b$. Then $a \hat{a} b$ which yields $a \varphi \sigma^{K} b \varphi$. The assertion now follows by Lemma 2.5. Let $\theta=$ $\mathscr{C}\left(\sigma^{K}, P^{\prime}, \tau^{\prime}\right)$.

6. $v K \theta$. By Lemma 2.6, we have

$$
\begin{aligned}
& \operatorname{ker} v=\operatorname{ker} \sigma \cup\left\{a \in P^{*} \mid a \varphi \in \operatorname{ker} \sigma\right\} \cup(\operatorname{ker} \tau)^{*}, \\
& \operatorname{ker} \theta=\operatorname{ker} \sigma^{K} \cup\left\{a \in P^{\prime *} \mid a \varphi \in \operatorname{ker} \sigma^{K}\right\} \cup\left(\operatorname{ker} \tau^{\prime}\right)^{*} .
\end{aligned}
$$

In order to prove that $\operatorname{ker} v=\operatorname{ker} \theta$, we let $a \in V$ and consider the following cases.

(i) $a \in S: a \in \operatorname{ker} v \Leftrightarrow a \in \operatorname{ker} \sigma \Leftrightarrow a \in \operatorname{ker} \sigma^{K} \Leftrightarrow a \in \operatorname{ker} \theta$.

(ii) $a \in P \backslash S: a \in \operatorname{ker} v \Leftrightarrow a \varphi \in \operatorname{ker} \sigma \Leftrightarrow a \varphi \in \operatorname{ker} \sigma^{K} \Leftrightarrow a \in \operatorname{ker} \theta$.

(iii) $a \in P^{\prime} \backslash P$ : If $a \in(\operatorname{ker} \tau)^{*}$, then ate from $e \in E(Q \backslash P)$ which by Lemma 2.5 yields

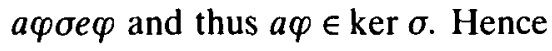

$$
a \in \operatorname{ker} v \Rightarrow a \in \operatorname{ker} \tau \Rightarrow a \varphi \in \operatorname{ker} \sigma \Rightarrow a \varphi \in \operatorname{ker} \sigma^{K} \Rightarrow a \in \operatorname{ker} \theta .
$$

For the converse, we first note that $a \in P^{\prime}$ implies $a \notin R$ and thus either $a \in \operatorname{ker} \tau$ or $a \varphi \notin \operatorname{ker} \sigma$. Hence the above sequence of implications can be reversed.

(iv) $a \in Q \backslash P^{\prime}$ : First note that

$$
\begin{aligned}
a \in \operatorname{ker} \theta & \Leftrightarrow a \in \operatorname{ker} \tau^{\prime} \\
& \Leftrightarrow a \in \operatorname{ker} \eta^{\kappa}, a \hat{\sigma} a^{2}, a \in \operatorname{ker} \zeta_{Q^{\prime} P^{\prime}} \\
& \Leftrightarrow a \in \operatorname{ker} \eta, a \varphi \sigma^{\kappa} a^{2} \varphi, a \in \operatorname{ker} \zeta_{Q^{\prime} P^{\prime}} \\
& \Leftrightarrow a \in \operatorname{ker} \tau, a \varphi \sigma a^{2} \varphi, a \in \operatorname{ker} \zeta_{Q^{\prime} P^{\prime}} \\
& \Leftrightarrow a \tau a^{2}, a \varphi \sigma a^{2} \varphi, a \zeta_{Q / P^{\prime}} a^{2} .
\end{aligned}
$$


Assume that $a \tau a^{2}$. By Lemma 2.5, we have $a \varphi \sigma a^{2} \varphi$. For any $x, y \in\left(Q / P^{\prime}\right)^{\prime}$, we have xay $\tau x a^{2} y$. By part $3, \tau$ saturates $Q \backslash P^{\prime}$ which then implies that $x a y \neq 0$ if and only if $x a^{2} y \neq 0$ in $Q / P^{\prime}$. Therefore $a \zeta_{Q / P} \cdot a^{2}$. Now (19) implies that

$$
a \in \operatorname{ker} v \Leftrightarrow a \in \operatorname{ker} \tau \Leftrightarrow a \in \operatorname{ker} \tau^{\prime} \Leftrightarrow a \in \operatorname{ker} \theta .
$$

Therefore ker $v=\operatorname{ker} \theta$.

7. If $v_{1} K v$, then $v_{1} \subseteq \theta$. Let $v_{1}=\mathscr{C}\left(\sigma_{1}, P_{1}, \tau_{1}\right)$ and assume that $v_{1} K v$. By Lemma 2.6, we have

$$
\operatorname{ker} v_{1}=\operatorname{ker} \sigma_{1} \cup\left\{a \in P_{1}^{*} \mid a \varphi \in \operatorname{ker} \sigma_{1}\right\} \cup\left(\operatorname{ker} \tau_{1}\right)^{*}
$$

which, together with (18), by hypothesis gives ker $\sigma_{1}=\operatorname{ker} \sigma$ and

$$
\left\{a \in P_{1}^{*} \mid a \varphi \in \operatorname{ker} \sigma_{1}\right\} \cup\left(\operatorname{ker} \tau_{1}\right)^{*}=\left\{a \in P^{*} \mid a \varphi \in \operatorname{ker} \sigma\right\} \cup(\operatorname{ker} \tau)^{*} .
$$

It follows that $\sigma_{1} K \sigma$ and hence $\sigma_{1} \subseteq \sigma^{K}$. In order to prove that $v_{1} \subseteq \theta$, by Lemma 2.3, it remains to show that

$$
P_{1} \subseteq P^{\prime}, \quad \tau_{1} \text { saturates } P^{\prime} \backslash P_{1},\left.\left.\quad \tau_{1}\right|_{Q \backslash P^{\prime}} \subseteq \tau^{\prime}\right|_{Q \backslash P^{\prime}}
$$

Let $a \in R \cap P_{1}$. Then $a \notin \operatorname{ker} \tau, a \varphi \in \operatorname{ker} \sigma$ and $a \in P_{1}^{*}$. The last two conditions imply that $a$ is in the left hand side of (20). But the first two conditions imply that $a$ is not in the right hand side of (20). This being impossible, we conclude that $R \cap P_{1}=\varnothing$. By Lemma 5.4, $P^{\prime}$ is the greatest ideal of $Q$ which is disjoint from $R$ and thus $P_{1} \subseteq P^{\prime}$. This establishes the first condition in (21).

In order to prove the second condition in (21), we let

$$
A=\left\{a \in Q \backslash P_{1} \mid a \tau_{1} b \text { for some } b \in P^{\prime} \backslash P_{1}\right\} \cup P_{1} .
$$

We show next that $A$ is an ideal of $Q$. Indeed, let $a \in A^{*}$ and $c \in Q \backslash P_{1}$ be such that $a c \notin P_{1}$. There exists $b \in P^{\prime} \backslash P_{1}$ such that $a \tau_{1} b$ by the definition of $A$. Hence $a c \tau_{1} b c$ whence $b c \notin P_{1}$ since $\tau_{1}$ is 0 -restricted. Thus $b c \in P^{\prime} \backslash P_{1}$ which yields $a c \in A^{*}$. By duality and since $P_{1}$ is an ideal of $Q$, we conclude that $A$ is an ideal of $Q$.

Now assume that $a \in A \cap R$. We have seen above that $P_{1} \cap R=\varnothing$. Hence $a \in A \backslash P_{1}$ and there exists $b \in P^{\prime} \backslash P_{1}$ such that $a \tau_{1} b$. Further, $a \in R$ implies that $a \notin \operatorname{ker} \tau$ and $a \varphi \in \operatorname{ker} \sigma$. Since $a \tau_{1} b$, by Lemma 2.5 we get $a \varphi \sigma_{1} b \varphi$; also $a \varphi \in \operatorname{ker} \sigma$ implies $a \varphi \in \operatorname{ker} \sigma_{1}$ whence $b \varphi \in \operatorname{ker} \sigma_{1}$. Hence $b \varphi \in \operatorname{ker} \sigma$ and since $b \in P^{\prime}$, we also have that $b \notin R$. But then $b \in \operatorname{ker} \tau$ by the definition of $R$. By (20), $a \notin \operatorname{ker} \tau$ implies $a \notin \operatorname{ker} \tau_{1}$, and $b \in \operatorname{ker} \tau$ implies that $b \in \operatorname{ker} \tau_{1}$. This is incompatible with $a \tau_{1} b$. Therefore $A \cap R=\varnothing$.

We have proved that $A$ is an ideal of $Q$ disjoint from $R$ which by Lemma 5.4 gives that $A \subseteq P^{\prime}$. It follows that $\tau_{1}$ saturates $P^{\prime} \backslash P_{1}$.

It remains to establish the last condition in (21). Since $\tau_{1}$ saturates both $Q \backslash P_{1}$ and $P^{\prime} \backslash P_{1}$, it also saturates $Q \backslash P^{\prime}$. Letting $\tau_{2}=\left(\left.\tau_{1}\right|_{Q \backslash P^{\prime}}\right) \cup\{(0,0)\}$, we get $\tau_{2} \in \mathscr{C}_{11}\left(Q / P^{\prime}\right)$. Now condition (20) yields that $\tau_{2} K \tau$ which implies that $\tau_{2} \subseteq \tau^{K}$. Let $a, b \in Q \backslash P^{\prime}$ be such that $a \tau_{1} b$. Then $a \tau_{2} b$ and thus $a \tau^{K} b$. Also $a \tau_{1} b$ implies that $a \varphi \sigma_{1} b \varphi$ whence $a \varphi \sigma^{K} b \varphi$ since $\sigma_{1} \subseteq \sigma^{K}$, and thus $a \hat{\sigma} b$. Since $\tau_{2} \in \mathscr{C}_{11}\left(Q / P^{\prime}\right)$ and $\zeta_{Q / p}$, is the greatest 0 -restricted congruence on $Q / P^{\prime}$, we get $\tau_{2} \subseteq \zeta_{Q / P^{\prime}}$. In particular, $a \zeta_{Q / P} \cdot b$. We have proved that $a \tau^{\prime} b$ which shows that $\left.\left.\tau_{1}\right|_{Q W^{\prime}} \subseteq \tau^{\prime}\right|_{Q \backslash P^{\prime}}$.

This completes the verification of the requirements in Lemma 2.3 for the inclusion $v_{1} \subseteq \theta$. Therefore $\theta$ has the required maximality so that $\theta=v^{K}$. 


\section{REFERENCES}

1. J. M. Howie, An introduction to semigroup theory (Academic Press, 1976).

2. F. Pastijn and M. Petrich, Congruences on regular semigroups, Trans. Amer. Math. Soc. 295 (1986), 607-633.

3. M. Petrich, Congruences on extensions of semigroups, Duke Math. J. 34 (1967), 215-224.

4. M. Petrich, The congruence lattice of an ideal extension of semigroups, Glasgow Math. J. 35 (1993), 39-50.

Department of Mathematics

UNIVERSITY OF WESTERN ONTARIO

LONDON, ONTARIO

Canada N6A 5B7 